\title{
A Distribuição Gama Weibull Poisson Aplicada a Dados de Sobrevivência
}

\author{
A. PERCONTINI ${ }^{1}{ }^{*}$, F.S. GOMES-SILVA ${ }^{1}$, M.W.A. RAMOS ${ }^{1}$, \\ R. VENANCIO ${ }^{1}$ e G.M. CORDEIRO ${ }^{2}$
}

Recebido em 16 outubro, 2012 / Aceito em 14 junho, 2014

\begin{abstract}
RESUMO. A família de distribuições univariadas gama-generalizada proposta por Zografos e Balakrishnan [3] foi discutida por Nadarajah et al. [7] que deram um amplo tratamento matemático a esta classe. Neste artigo, estudamos o modelo Gama Weibull Poisson, que tem como casos especiais, várias distribuições discutidas na literatura. Deduzimos uma expressão explícita para a entropia de Rényi, e mostramos que a densidade da distribuição Gama Weibull Poisson é uma mistura de densidades da distribuição Weibull Poisson. Estudamos algumas propriedades matemáticas importantes como momentos, função geratriz de momentos e função quantílica.
\end{abstract}

Palavras-chave: Gama Weibull Poisson, máxima verossimilhança, taxa de risco.

\section{INTRODUÇÃO}

Inicialmente o estudo da distribuição Weibull [9] lidou com resistência dos materiais e foi publicado em um jornal escandinavo. Posteriormente, um artigo publicado por Weibull [10] modelando conjuntos de dados de áreas diferentes mostrou a versatilidade do modelo em termos de suas aplicações a diferentes áreas. Um relatório da distribuição Weibull [11] lista mais de 1000 referências para suas aplicações e outra pesquisa indica que este número tem aumentado ao longo dos anos. A distribuição Weibull tem sido amplamente usada para analisar dados de tempo de vida. Ao modelar taxa de risco monótona, a distribuição Weibull é uma opção de escolha pois as formas de sua densidade são positivamente e negativamente assimétricas. Entretanto, ela não apresenta um ajuste paramétrico que se adeque para modelar fenômenos que têm taxa de risco não-monótona tais como, as que têm forma de "banheira" e as taxas de risco unimodais, muito usadas em estudos biológicos. Nos últimos anos, inúmeros modelos deduzidos ou de alguma forma relacionados com a distribuição Weibull têm sido propostos. Uma característica dessas

\footnotetext{
*Autor correspondente: Ana Carla Percontini.

${ }^{1}$ PPGMC, Universidade Federal de Pernambuco, 50740-530 Recife, PE, Brasil. E-mails: anappaixao@ gmail.com; franksinatrags@gmail.com; wallace.ifpb@gmail.com; ronaldovenanciorvs@gmail.com

${ }^{2}$ Departamento de Estatística, Universidade Federal de Pernambuco, 50740-530 Recife, PE, Brasil.

E-mail: gausscordeiro@gmail.com
} 
novas distribuições é apresentar função de taxa de risco em forma de $U$, as quais são muito úteis em análise de sobrevivência.

Neste artigo, estudamos uma nova distribuição denominada Gama Weibull Poisson (GWP) que é a composição da distribuição Gama com a distribuição Weibull Poisson (WP) introduzida por Lu e Shi [8]. Esta distribuição também foi estudada por Morais e Barrato-Souza [1], como um caso especial da classe de distribuições Weibull Power Series (WPS).

Este artigo é organizado como segue. Na Seção 2, definimos o novo modelo e discutimos alguns de seus casos especiais. Na Seção 3, deduzimos algumas propriedades da GWP, tais como, a taxa de risco, expansão da densidade GWP como uma mistura de densidades WP, função de sobrevivência, momentos, função geratriz de momentos (fgm), função quantílica e entropia de Rényi. A estimação de máxima verossimilhança de um modelo paramétrico é descrita na Seção 4. Na Seção 5, fazemos uma aplicação do modelo GWP a um conjunto de dados reais. Conclusões e observações são dadas na Seção 6.

Neste artigo os resultados numéricos apresentados foram obtidos utilizando o ambiente de programação e análise de dados $\mathrm{R}^{3}$ em sua versão 2.15.1, bem como, o Maple $14^{4}$ para os cálculos do $n$-ésimo momento e das derivadas da log-verossimilhança.

\section{A DISTRIBUIÇÃO GAMA WEIBULL POISSON}

Zografos e Balakrishnan [3] e Ristic e Balakrishnan [4] propuseram diferentes famílias de distribuições univariadas geradas a partir da distribuição gama. A família de Zografos e Balakrishnan [3], chamada Gama- $G$, é definida da seguinte forma, se $G(x)$ é uma função de distribuição acumulada (fda) de uma variável aleatória contínua, então, a fda da função geradora gama de distribuições é dada por

$$
F(x)=\frac{\gamma(a,-\log [1-G(x)])}{\Gamma(a)}=\frac{1}{\Gamma(a)} \int_{0}^{-\log [1-G(x)]} t^{a-1} \exp (-t) d t,
$$

em que $a>0$ é um parâmetro de forma. Dessa forma, derivando a equação (2.1), obtém-se a sua função densidade de probabilidade (fdp) expressa como

$$
f(x)=\frac{1}{\Gamma(a)}\{-\log [1-G(x)]\}^{a-1} g(x),
$$

em que as funções $\Gamma(a)=\int_{0}^{\infty} t^{a-1} \exp (-t) d t$ e $\gamma(a, z)=\int_{0}^{z} t^{a-1} \exp (-t) d t$ denotam a função gama e a função gama incompleta inferior, respectivamente, e $g(x)=d G(x) / d x$. O parâmetro de forma $a$ flexibiliza a distribuição a ser modificada e o suporte de $f(x)$ é definido por $g(x)$. A distribuição GWP segue a família Gama- $G$ em que G é a fda da distribuição WP.

Sejam $\left\{W_{i}\right\}_{i=1}^{Z}$ variáveis aleatórias independentes e indenticamente distribuídas tendo uma função de densidade Weibull definida por

$$
\pi(w ; \alpha, \beta)=\alpha \beta w^{\alpha-1} \exp \left(-\beta w^{\alpha}\right), w>0,
$$

\footnotetext{
${ }^{3}$ http://www.r-project.org/

${ }^{4}$ http://www.maplesoft.com
} 
onde $\alpha>0$ é o parâmetro de forma e $\beta>0$ o parâmetro de escala. Supomos que $Z$ tem uma distribuição Poisson truncada com parâmetro $\lambda>0$ e função de probabilidade de massa dada por

$$
p(z, \lambda)=\mathrm{e}^{-\lambda} \lambda^{z} \Gamma^{-1}(z+1)\left(1-\mathrm{e}^{-\lambda}\right)^{-1}, z=1,2, \ldots
$$

Seja $X_{Z}=\min \left\{W_{1}, W_{2}, \ldots, W_{Z}\right\}$, em que a variável aleatória $Z$ e os W's são supostos independentes. A distribuição Weibull Poisson para $X_{Z}$ tem função de densidade dada por

$$
g(x ; \alpha, \beta, \lambda)=\dot{c} x^{\alpha-1} u \mathrm{e}^{\lambda u}, x>0,
$$

sendo $\dot{c}=\dot{c}(\alpha, \beta, \lambda)=\frac{\alpha \beta \lambda \mathrm{e}^{-\lambda}}{1-\mathrm{e}^{-\lambda}}$ e $u=\mathrm{e}^{-\beta x^{\alpha}}$. A expressão (2.3) é a equação (1), encontrada em Lu e Shi [8]. A fda correspondente a esta expressão é

$$
G(x)=\frac{\mathrm{e}^{\lambda u}-\mathrm{e}^{\lambda}}{1-\mathrm{e}^{\lambda}}, \quad x>0 .
$$

Deduzimos uma expressão para a fdp da distribuição GWP inserindo (2.3) e (2.4) em (2.2). Logo,

$$
f(x ; \boldsymbol{\theta})=c x^{\alpha-1} u \mathrm{e}^{\lambda u}\left[\log \left(\frac{1-\mathrm{e}^{\lambda}}{1-\mathrm{e}^{\lambda u}}\right)\right]^{a-1},
$$

em que $c=\frac{\alpha \beta \lambda \mathrm{e}^{-\lambda}}{\Gamma(a)\left(1-\mathrm{e}^{-\lambda}\right)}, a>0$ e $\boldsymbol{\theta}=(a, \alpha, \beta, \lambda)$.

Se $G(x)$ é a fda da distribuição WP, então a equação (2.1) fornece a fda da distribuição proposta GWP dada por

$$
F(x)=1-\frac{\Gamma\left(a, \log \left[\frac{1-e^{\lambda}}{1-e^{\lambda u}}\right]\right)}{\Gamma(a)},
$$

em que $\Gamma(a, x)=\int_{x}^{\infty} t^{a-1} e^{-t} d t$ é a função gama incompleta superior.

A função de sobrevivência é definida como $S(t)=1-F(t)$, e especifica a probabilidade de um certo elemento sobreviver pelo menos até o tempo $t$. Dessa forma, a função de sobrevivência de uma variável aleatória com distribuição GWP é dada por

$$
S(x)=\frac{\Gamma\left(a, \log \left[\frac{1-e^{\lambda}}{1-e^{\lambda u}}\right]\right)}{\Gamma(a)} .
$$

A variável aleatória $X$ que tem função de densidade (2.5) é denotada por $X \sim \operatorname{GWP}(a, \alpha, \beta, \lambda)$, em que $a, \alpha>0$ são parâmetros de forma, $\beta>0$ é o parâmetro de escala e $\lambda>0$. Gráficos da equação (2.5) para algumas escolhas dos parâmetros são apresentados na Figura 1. Além disso, podemos verificar que a distribuição GWP possui vários submodelos bem conhecidos como casos especiais, descritos a seguir:

- Para $\alpha=1$ e $\alpha=2$, a Equação (2.5) se reduz a Gama Exponencial Poisson (GEP) e Gama Rayleigh Poisson (GRP), respectivamente; 
- Fazendo $\lambda \rightarrow 0$, obtemos na Equação (2.5) a distribuição GW. Adicionalmente, quando $\alpha=1$ e $\alpha=2$ obtemos as distribuições Gama Exponencial (GE) e Gama Rayleigh (GR), respectivamente;

- Se $a=1$, a Equação (2.5) se reduz a distribuição WP. Adicionalmente, se $\alpha=1$ e $\alpha=2$, obtemos as distribuições Exponencial Poisson (EP) e Rayleigh Poisson (RP), nesta ordem;

- Obtemos a distribuição half-normal (HN) fazendo $a=3$ e $\alpha=2$ em adição a $\lambda \rightarrow 0$;

- Fazendo $\alpha=2$ na Equação (2.5), a distribuição log-normal (LN) é um caso limite especial quando $a \rightarrow \infty$ e $\lambda \rightarrow 0$;

- Se $a=1$ e $\lambda \rightarrow 0$, a Equação (2.5) se reduz a distribuição Weibull (W). Adicionalmente, fazendo $\alpha=1$ e $\alpha=2$, obtemos as distribuições Exponencial (E) e Rayleigh (R), respectivamente;

- Se $\lambda \rightarrow 0, \beta=1 / 2$ e $a=n / 2$ obtemos a distribuição $\chi_{n}^{2}$ com $n$ graus de liberdade;

- Fazendo $\lambda \rightarrow 0, a=v-1, \beta=\phi^{-1}$ e $\alpha=1$, a Equação (2.5) se reduz a distribuição Maxwell (M);

- Se $\lambda \rightarrow 0$ e $\alpha=1$, em que $a=0,1,2, \ldots$, obtemos a distribuição Erlang.

Estamos motivados em estudar o modelo GWP por causa da ampla utilização da distribuição Weibull. Adicionado a este fato, a generalização estudada neste artigo estabelece uma extensão da Weibull a situações ainda mais complexas. Um outro ponto positivo é que a distribuição WP constitui um exemplar básico da família proposta.

\section{PROPRIEDADES DA DISTRIBUIÇÃO GWP}

Nesta seção, apresentamos propriedades matemáticas da distribuição GWP: funções de risco, momentos, função geratriz de momentos, função quantílica, entropia de Rényi. Além disso, provamos que a densidade da GWP é uma mistura de densidades WP.

\subsection{Função taxa de risco}

A taxa de risco da distribuição GWP é dada por

$$
h(x ; \boldsymbol{\theta})=\frac{c \Gamma(a) x^{\alpha-1} u e^{\lambda u}\left[\log \left(\frac{1-e^{\lambda}}{1-e^{\lambda u}}\right)\right]^{a-1}}{\Gamma\left(a, \log \left[\frac{1-e^{\lambda}}{1-e^{\lambda u}}\right]\right)} .
$$

Na Figura 2 temos as possíveis formas da taxa de risco da distibuição GWP. A função de risco é mais informativa do que a função de sobrevivência. Funções de sobrevivência podem ter formas semelhantes, enquanto as respectivas funções de risco podem diferir de maneira drástica. A GWP apresenta função de risco crescente, decrescente e na forma de banheira invertida ou unimodal. 
(a)

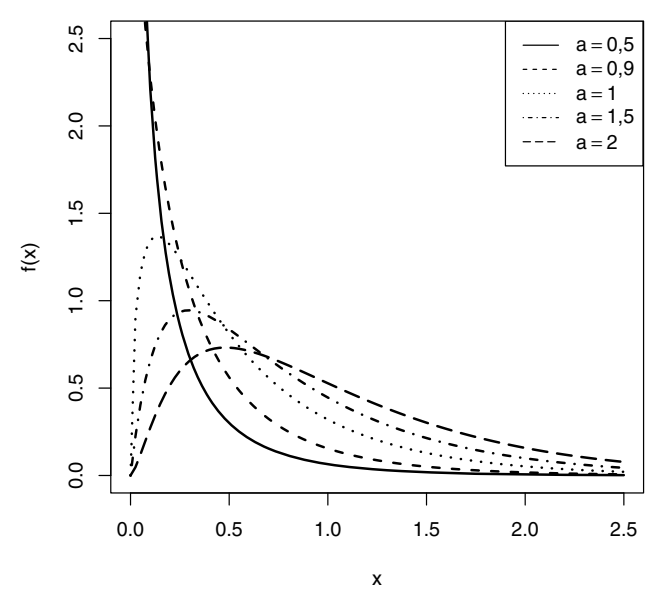

(c)

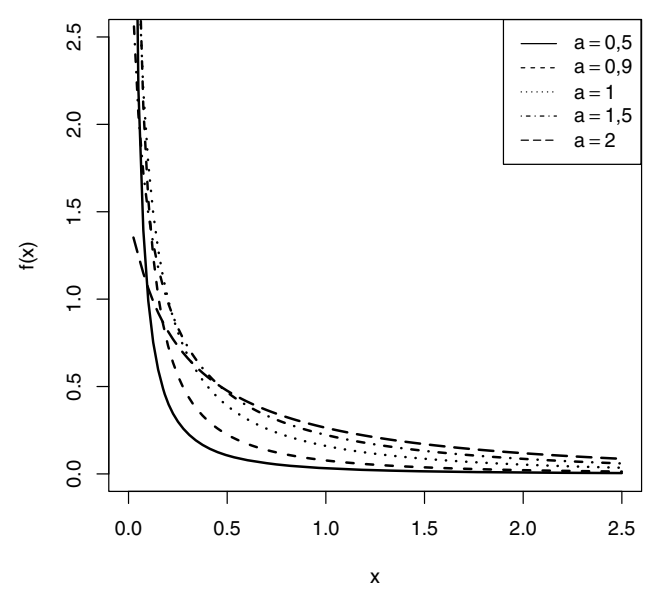

(b)

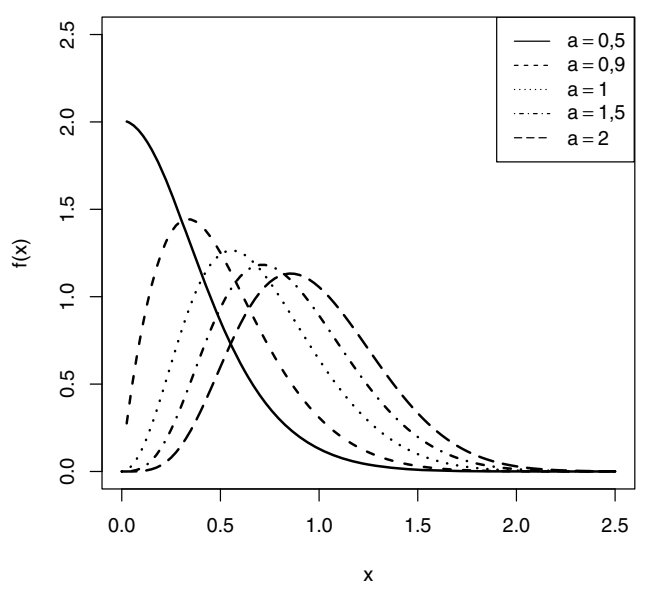

(d)

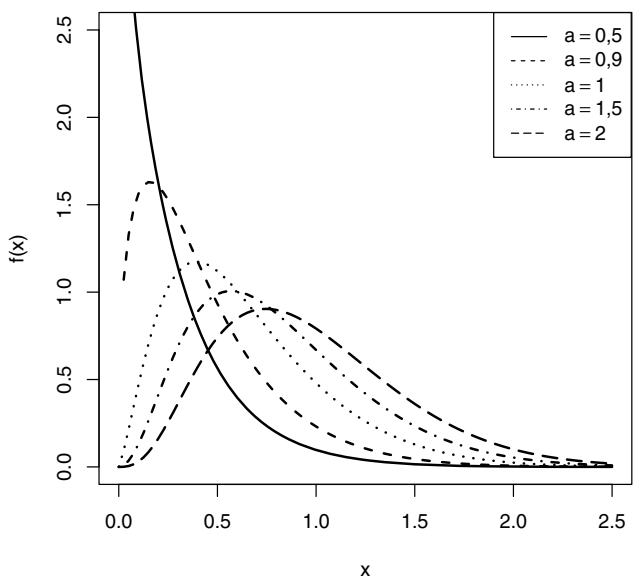

Figura 1: Gráficos de densidades da distribuição GWP.

\subsection{Expansão da densidade}

As Equações (2.5) e (2.6) podem ser expressas em termos de distribuições exponencializadas. Considere $G(x)$ uma função de distribuição de uma variável aleatória $X$ e $g(x)$ a fdp associada. Se $X$ segue uma distribuição $G$-exponencializada com parâmetro $a$, isto é, $X \sim \exp -G(a)$, então sua fdp e fda são dadas, respectivamente, por

$$
h_{a}=a G^{a-1}(x) g(x)
$$

e

$$
H_{a}=G^{a}(x), \quad x>0 .
$$


(a)

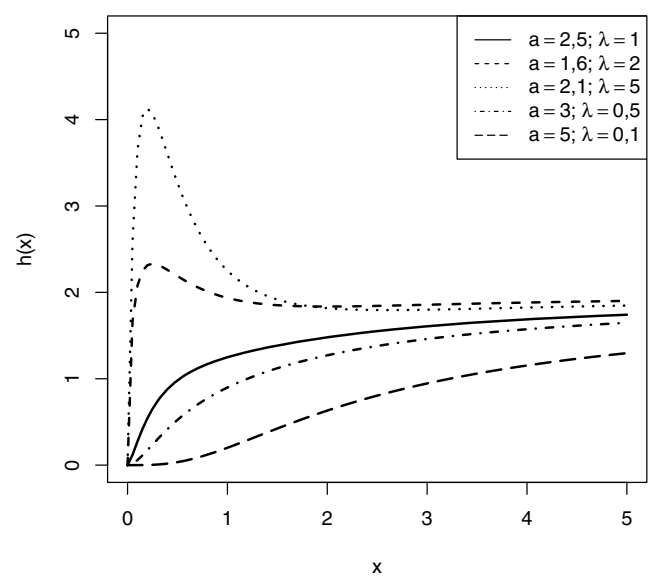

(b)

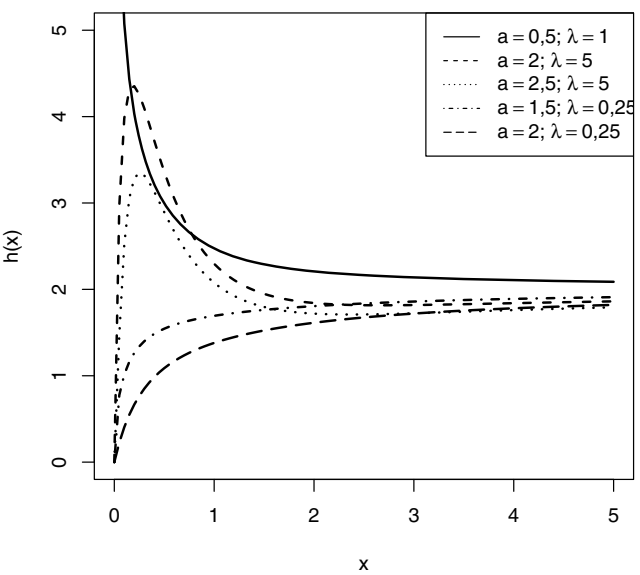

Figura 2: Taxa de Risco para: $\alpha=1, \beta=2$

As expansões para a fdp $f(x)$ e fda $F(x)$ da Gama- $G$ são apresentadas em Nadarajah et al. [7] como

$$
f(x)=\sum_{k=0}^{\infty} b_{k} h_{a+k}(x)
$$

$\mathrm{e}$

$$
F(x)=\sum_{k=0}^{\infty} b_{k} H_{a+k}(x)
$$

respectivamente, em que

$$
b_{k}=\frac{\left(\begin{array}{c}
k+1 \\
k
\end{array}\right)}{(a+k) \Gamma(a-1)} \sum_{j=0}^{k} \frac{(-1)^{j+k}\left(\begin{array}{l}
k \\
j
\end{array}\right) p_{j, k}}{(a-1-j)} .
$$

A constante $p_{j, k}$ pode ser calculada recursivamente por

$$
p_{j, k}=k^{-1} \sum_{m=1}^{k}[k-m(j+1)] c_{m} p_{j, k-m},
$$

para $k=1,2, \ldots$ com $p_{j, 0}=1$ e $c_{k}=(-1)^{k+1}(k+1)^{-1}$.

Substituindo as expressões (2.3), (2.4) e (3.1) em (3.3), obtemos a densidade da distribuição GWP como uma mistura de densidades WP, expressa por

$$
f(x)=\sum_{k=0}^{\infty} \sum_{i=0}^{a+k-1} b_{k, i} g\left(x ; \alpha, \beta, \lambda_{k, i}\right),
$$

em que $\lambda_{k, i}=\lambda *(a+k-i)$ e $b_{k, i}=\frac{\lambda(a+k) b_{k}(-1)^{i} \mathrm{e}^{\lambda(i-1)}\left(\begin{array}{c}a+k-1 \\ i\end{array}\right)\left(1-\mathrm{e}^{-\lambda_{k, i}}\right)}{\lambda_{k, i}\left(1-\mathrm{e}^{-\lambda}\right) \mathrm{e}^{-\lambda_{k, i}}}$. 
Usando a Equação (3.4), após algumas manipulações algébricas, a fda da distribuição GWP pode ser expressa como

$$
F(x)=\sum_{k=0}^{\infty} \sum_{i=0}^{a+k-1} b_{k, i} G\left(x ; \alpha, \beta, \lambda_{k, i}\right)
$$

\subsection{Momentos da Distribuição GWP}

De agora em diante, seja $X \sim \operatorname{GWP}(a, \alpha, \beta, \lambda)$. Usando a Equação (3.6), podemos obter o $n$-ésimo momento de $X$ como

$$
E\left(X^{n}\right)=\sum_{k=0}^{\infty} \sum_{i=0}^{a+k-1} b_{k, i} \int_{0}^{\infty} x^{n} g\left(x ; \alpha, \beta, \lambda_{k, i}\right) d x,
$$

em que $g\left(x ; \alpha, \beta, \lambda_{k, i}\right)$ é a fdp da distribuição WP dada em (2.3). Portanto,

$$
E\left(X^{n}\right)=\sum_{k=0}^{\infty} \sum_{i=0}^{a+k-1} \frac{b_{k, i}}{e^{\lambda_{k, i}}-1} \int_{0}^{\lambda_{k, i}} e^{x}\left[-\beta^{-1}\left(\log x-\log \lambda_{k, i}\right)\right]^{\frac{n}{\alpha}} d x
$$

\subsection{Função Geratriz de Momentos}

A função geratriz de momentos, fgm, de $X$ é dada por

$$
M(t)=E\left(e^{t X}\right)=\sum_{n=0}^{\infty} \frac{t^{n} E\left(X^{n}\right)}{n !}
$$

em que $E\left(X^{n}\right)$ é obtido em (3.7). Dessa forma temos que

$$
M(t)=\sum_{n=0}^{\infty} w_{n} \int_{0}^{\lambda_{k, i}} e^{x}\left[-\beta^{-1}\left(\log x-\log \lambda_{k, i}\right)\right]^{\frac{n}{\alpha}} d x
$$

em que

$$
w_{n}=\sum_{k=0}^{\infty} \sum_{i=0}^{a+k-1} \frac{t^{n} b_{k, i}}{n !\left(e^{\lambda_{k, i}}-1\right)}
$$

\subsection{Função Quantílica}

Nadarajah et al. [7], demonstraram que a função quantílica da distribuição Gama- $G$ é escrita como

$$
F^{-1}(u)=G^{-1}\left\{1-\mathrm{e}^{-Q^{-1}(a, 1-u)}\right\}, 0<u<1,
$$

em que $Q^{-1}(a, u)$ é a função inversa de $Q(a, x)=1-\gamma(a, x) / \Gamma(a, x)$.

A partir da função quantílica da distribuição WP encontrada em Lu e Shi [8], juntamente com a Equação (3.9), obtemos a função quantílica da distribuição GWP, como

$$
F^{-1}(u)=\left\{-\beta^{-1} \log \left[\lambda^{-1} \log \left[\left(1-e^{-Q^{-1}(a, 1-u)}\right)\left(1-e^{\lambda}\right)+e^{\lambda}\right]\right]\right\}^{\frac{1}{\alpha}}, \quad 0<u<1 .
$$


A função $Q^{-1}(a, u)$ admite uma expansão em série de potência dada por

$$
Q^{-1}(a, u)=\sum_{i=0}^{\infty} m_{i}[\Gamma(a+1) u]^{i / a},
$$

em que $m_{0}=0, m_{1}=1$ e qualquer coeficiente $m_{i+1}$ para $i \geq 1$, é determinado pela equação de recorrência

$$
\begin{aligned}
m_{i+1}= & \frac{1}{i(a+i)}\left\{\sum_{r=1}^{i} \sum_{s=1}^{i-s+1} m_{r} m_{s} m_{i-r-s+2} s(i-r-s+2)\right. \\
& \left.-\Delta(i) \sum_{r=2}^{i} m_{r} m_{i-r+2} r[r-a-(1-a)(i+2-r)]\right\},
\end{aligned}
$$

em que $\Delta(i)=0$ se $i<2$ e $\Delta(i)=1$ se $i \geq 2$.

\subsection{Entropia de Rényi}

A entropia de Rényi é uma medida de variação de incerteza que tem sido usada em aplicações e caracterizações de muitas distribuições de probabilidade. Nessa seção, deduzimos uma expressão para esta medida. Para a função de densidade $f(x)$, a entropia de Rényi é definida por

$$
I_{R}(\varphi)=(1-\varphi)^{-1} \log \left[\int_{0}^{\infty} f(t)^{\varphi} d t\right]
$$

em que $\varphi>0$ e $\varphi \neq 1$.

De acordo com Nadarajah et al. [7], a entropia da distribuição Gama- $G$ é dada pela função

$$
I_{R}(\gamma)=-\frac{\gamma \log \Gamma(a)}{1-\gamma}+\frac{1}{1-\gamma} \log \left\{\sum_{k=0}^{\infty}\left(\begin{array}{c}
k-\gamma a+\gamma \\
k
\end{array}\right) \sum_{j=0}^{k} \frac{(-1)^{j+k}\left(\begin{array}{l}
k \\
j
\end{array}\right) p_{j, k}}{[\gamma(a-1)-j]} I_{k}\right\}
$$

em que

$$
I_{k}=\int_{0}^{\infty} G(x)^{[\gamma(a-1)+k]} g^{\gamma}(x) d x .
$$

Aplicando (2.3) e (2.4) em (3.10) temos que a entropia de Rényi da distribuição GWP pode ser escrita como

$$
I_{R}(\gamma)=-\frac{\gamma \log \Gamma(a)}{1-\gamma}+\frac{1}{1-\gamma} \log \left\{\sum_{\gamma, n, k=0}^{\infty} \sum_{j=0}^{k} \sum_{i=0}^{\gamma a-\gamma+k} \omega_{(r, n, k, j, i)} p_{j, k} \Gamma\left(\gamma-\frac{\gamma}{\alpha}+r-\frac{1}{\alpha}\right)\right\},
$$

em que

$$
\omega_{(r, n, k, j, i)}=\frac{(-1)^{r+k+j+i} \mathrm{e}^{\lambda i}(\gamma \beta)^{r}[\lambda(\gamma a+k-i)]^{n}\left(\begin{array}{c}
k \\
j
\end{array}\right)\left(\begin{array}{c}
\gamma a-\gamma+k \\
i
\end{array}\right)\left(\begin{array}{c}
k-\gamma a+\gamma \\
k
\end{array}\right)}{(\gamma a-\gamma-j)\left(1-\mathrm{e}^{\lambda}\right)^{\gamma a-\gamma+k}} .
$$




\section{LOG-VEROSSIMILHANÇA}

Nesta seção examinamos a estimativa de máxima verossimilhança (EMV) de $\boldsymbol{\theta}$ para a distribuição GWP. Sejam $X_{1}, X_{2}, \ldots, X_{n}$ uma amostra aleatória de $X$ com valores observados $x_{1}, x_{2}, \ldots, x_{n}$ e seja $\theta=(a, \alpha, \beta, \lambda)^{T}$ o vetor dos parâmetros do modelo. A função de logverossimilhança para $\boldsymbol{\theta}$ reduz-se a

$$
\begin{aligned}
l(\boldsymbol{\theta})= & n \log (c)+(\alpha-1) \sum_{i=1}^{n} \log \left(x_{i}\right)+\sum_{i=1}^{n} \log \left(u_{i}\right) \\
& +\lambda \sum_{i=1}^{n} \log \left(u_{i}\right)+(a-1) \sum_{i=1}^{n} \log \left[\log \left(\frac{1-e^{\lambda}}{1-e^{\lambda u_{i}}}\right)\right],
\end{aligned}
$$

e, então,

$$
\begin{aligned}
\frac{\partial l}{\partial \alpha}= & \sum_{i=1}^{n} \log \left(x_{i}\right)-\beta(1+\lambda) \sum_{i=1}^{n} x_{i}^{\alpha} \log \left(x_{i}\right) \\
& -\lambda u_{\beta}(a-1) \sum_{i=1}^{n} \frac{x_{i}^{\alpha} u_{i} e^{\lambda u_{i}} \log \left(u_{i}\right)}{1-e^{\lambda u_{i}}} \log ^{-1}\left(\frac{1-e^{\lambda}}{1-e^{\lambda u_{i}}}\right), \\
\frac{\partial l}{\partial \beta}= & -\sum_{i=1}^{n} x_{i}^{\alpha}-\lambda \sum_{i=1}^{n} x_{i}^{\alpha}-\lambda(a-1) \sum_{i=1}^{n} \frac{x_{i}^{\alpha} u_{i} e^{\lambda u_{i}}}{1-e^{\lambda u_{i}}} \log ^{-1}\left(\frac{1-e^{\lambda}}{1-e^{\lambda u_{i}}}\right), \\
\frac{\partial l}{\partial \lambda}= & -\sum_{i=1}^{n} \log \left(u_{i}\right)+\sum_{i=1}^{n}\left[\frac{-e^{\lambda}}{1-e^{\lambda}}+\frac{u_{i} e^{\lambda u_{i}}}{1-e^{\lambda u_{i}}}\right] \log ^{-1}\left(\frac{1-e^{\lambda}}{1-e^{\lambda u_{i}}}\right) \quad \mathrm{e} \\
\frac{\partial l}{\partial a}= & \sum_{i=1}^{n} \log \left[\log \left(\frac{1-e^{\lambda}}{1-e^{\lambda u_{i}}}\right)\right]
\end{aligned}
$$

O sistema de equações anterior não admite forma fechada sendo necessário métodos numéricos para resolvê-lo. As estimativas dos parâmetros são obtidas através da maximização numérica do logaritmo da função de verossimilhança por meio do algoritmo de otimização não linear quasiNewton conhecido como BFGS.

O teste da razão de verossimilhanças (RV) pode ser usado para comparar o modelo GWP com seus submodelos. Podemos calcular os valores de máximo da log-verossimilhança restrita e irrestrita para construir a estatística da RV para testar submodelos da distribuição GWP. Por exemplo, o teste $H_{0}: a=1$ versus $H_{1}: H_{0}$ não é verdade, é equivalente a comparar as distribuições GWP e WP. A estatística da RV é definida como

$$
w=2\{\ell(\hat{a}, \hat{\alpha}, \hat{\beta}, \hat{\lambda})-\ell(\tilde{1}, \tilde{\alpha}, \tilde{\beta}, \tilde{\lambda})\}
$$

em que $\hat{a}, \hat{\alpha}, \hat{\beta}$ e $\hat{\lambda}$ são as EMVs sob $H_{1}$ e $\tilde{\alpha}, \tilde{\beta}$ e $\tilde{\lambda}$ são as estimativas sob $H_{0}$. Rejeita-se a hipótese nula quando $w>\chi_{1-\gamma}^{2}(1)$, que é o quantil $(1-\gamma)$ da distribuição qui-quadrado com um grau de liberdade. 


\section{APLICAÇÃO}

Ao longo desta seção vamos ajustar os modelos de distribuição GWP, WP e W a um conjunto de dados reais. Os dados de manutenção que seguem, representam 46 observações do tempo ativo de reparo, em horas, de um transceptor de comunicação aéreo encontrados em [12], [6], [8] e [2]: 0,$2 ; 0,3 ; 0,5 ; 0,5 ; 0,5 ; 0,5 ; 0,6 ; 0,6 ; 0,7 ; 0,7 ; 0,7 ; 0,8 ; 0,8 ; 1,0 ; 1,0 ; 1,0 ; 1,0 ; 1,1 ; 1,3 ; 1,5 ; 1,5$; 1,$5 ; 1,5 ; 2,0 ; 2,0 ; 2,2 ; 2,5 ; 2,7 ; 3,0 ; 3,0 ; 3,3 ; 3,3 ; 4,0 ; 4,0 ; 4,5 ; 4,7 ; 5,0 ; 5,4 ; 5,4 ; 7,0 ; 7,5 ; 8,8$; 9,$0 ; 10,3 ; 22,0$ e 24,5 .

A Tabela 1 lista as estimativas de máxima verossimilhança e seus correspondentes erros-padrão dos modelos paramétricos. Os critérios da informação de Akaike (AIC), Bayesiano (BIC) e Akaike Corrigido (CAIC) são utilizados para a seleção dos modelos. Ajustamos a distribuição Beta Weibull Poisson (BWP), recentemente introduzida por Percontini et al. [2], para fazer uma comparação com o modelo GWP. A BWP é um modelo da família de distribuições beta- $G$ [5], competitivo ao nosso, possuindo cinco parâmetros, cuja fdp é dada por

$$
f(x)=c x^{\alpha-1} u \mathrm{e}^{\lambda u}\left(\mathrm{e}^{\lambda}-\mathrm{e}^{\lambda u}\right)^{p}\left(\mathrm{e}^{\lambda u}-1\right)^{q-1}\left(\mathrm{e}^{\lambda u}-\mathrm{e}^{\lambda}\right)^{-1},
$$

em que

$$
c=\frac{\alpha \beta \lambda \mathrm{e}^{-\lambda}\left(\mathrm{e}^{\lambda}-1\right)^{2-p-q}}{B(p, q)\left(\mathrm{e}^{-\lambda}-1\right)}, \quad p>0 \text { e } q>0
$$

são parâmetros de forma adicionais e $B(p, q)=\frac{\Gamma(p) \Gamma(q)}{\Gamma(p+q)}$ é a função beta. Os resultados indicam que o modelo GWP apresenta os menores valores das estatísticas AIC, BIC e CAIC, sugerindo que ele tem um melhor ajuste para este conjunto de dados dentre os modelos ajustados.

Tabela 1: EMVs dos parâmetros e estatísticas AIC, BIC, CAIC dos modelos GWP, WP e W para os dados de tempo ativo de reparo de um transceptor de comunicação aéreo.

\begin{tabular}{|c|c|c|c|c|c|c|c|c|}
\hline Modelo & & $\widehat{a}$ & $\widehat{\alpha}$ & $\widehat{\beta}$ & $\widehat{\lambda}$ & AIC & BIC & CAIC \\
\hline GWP & & 14,145 & 0,644 & 1,591 & 15,115 & 206,133 & 213,447 & 207,108 \\
& & $(12,891)$ & $(0,053)$ & $(0,885)$ & $(12,405)$ & & & \\
WP & & & & & & & & \\
& & & 1,101 & 0,092 & 3,522 & 210,927 & 216,413 & 211,499 \\
& & & $(0,120)$ & $(0,052)$ & $(1,917)$ & & & \\
W & & & 0,899 & 0,334 & & 212,939 & 216,597 & 213,218 \\
& & & $(0,096)$ & $(0,075)$ & & & & \\
\hline \hline Modelo & $\widehat{p}$ & $\widehat{q}$ & $\widehat{\alpha}$ & $\widehat{\beta}$ & $\widehat{\lambda}$ & AIC & BIC & CAIC \\
\hline BWP & 21,474 & 0,320 & 0,724 & 1,428 & 5,340 & 207,838 & 216,982 & 209,339 \\
& $(56,050)$ & $(0,256)$ & $(0,386)$ & $(1,385)$ & $(2,235)$ & & & \\
\hline
\end{tabular}

A estatística RV para testar a hípótese nula $H_{0}$ : WP contra a hipótese alternativa $H_{1}$ : GWP é 6,7954 (p-valor $=9,13 \times 10^{-3}$ ). Portanto, para qualquer nível de significância usual, rejeitamos a hipótese nula em favor da distribuição GWP. Os gráficos das densidades dos modelos ajustados são dados na Figura 3. 


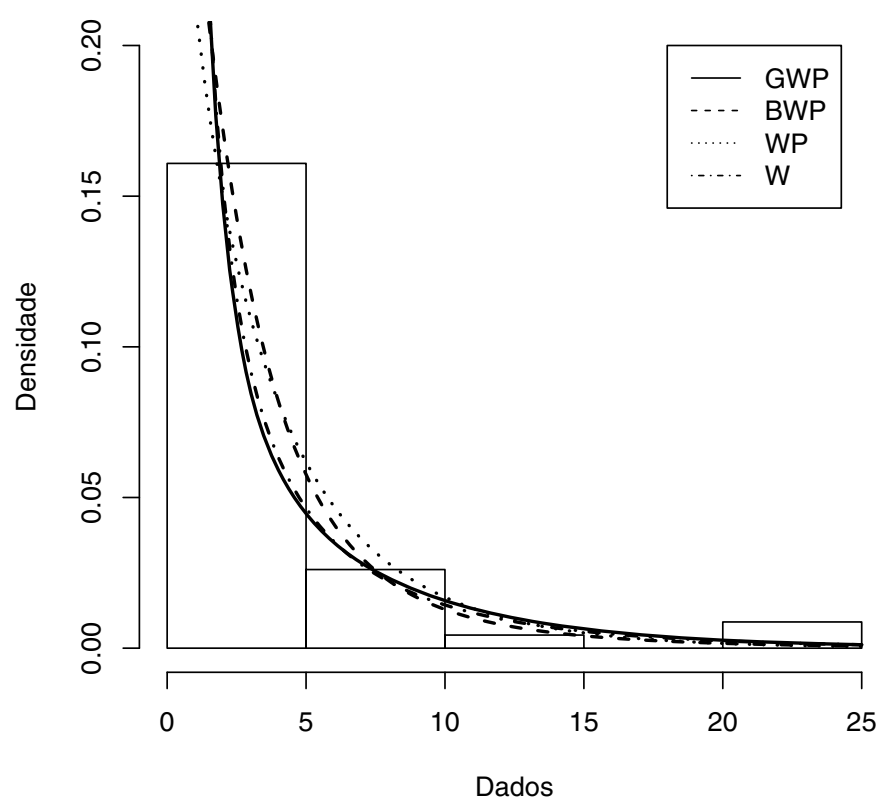

Figura 3: Gráficos das densidades dos modelos ajustados

\title{
6 CONCLUSÃO
}

A distribuição GWP é flexível na análise dos dados comparativamente a outros modelos de sobrevivência. A sua função densidade de probabilidade pode ser expressa como uma mistura de densidades Weibull Poisson. Apresentamos um tratamento matemático da distribuição obtendo expansões da função de densidade, função acumulada, função geratriz de momentos, função quantílica e entropia de Rényi. A estimação dos parâmetros do modelo é realizada por máxima verossimilhança. Uma aplicação a dados reais revela que a distribuição GWP pode fornecer um melhor modelo de sobrevivência que os modelos W, WP e BWP.

\begin{abstract}
In this paper, we study the gamma Weibull Poisson distribution that has many special cases of distributions discussed in the literature. We study some important mathematical properties such as moments, moment generating and quantile functions. We obtain an explicit expression for Rényi entropy. Further, we write the probability density function of the GWP distribution as a linear combination of WP density functions.
\end{abstract}

Keywords: Gamma Weibull Poisson distribution, maximum likelihood, hazard rate.

\section{REFERÊNCIAS}

[1] A. Morais \& W. Barrato-Souza. A compound class of Weibull and power series distribution. Comput. Statist. Data Anal., 55 (2011), 1410-1425. 
[2] A. Percontini, B. Blas \& G. M. Cordeiro. The beta Weibull Poisson distribution. Chilean Journal of Statistics, 4 (2013), 3-26.

[3] K. Zografos \& N. Balakrishnan. On families of beta and generalized gamma-generated distributions and associated inference. Statistical Methodology, 6 (2009), 344-362.

[4] M. Ristic \& N. Balakrishnan. The gamma exponentiated exponential distribution. Journal of Statistical Computation an Simulation, 82 (2012), 1191-1206.

[5] N. Eugene, C. Lee \& F. Famoye. Beta-normal distribution and its applications. Communication in Statistics - Theory and Methods, 31 (2002), 497-512.

[6] R.S. Chhikara \& J.L. Folks. The inverse Gaussian distribution as a lifetime model. Technometrics, 19 (1977), 461-468.

[7] S. Nadarajah, G.M. Cordeiro \& E.M. Ortega. The gamma-G family of distributions: Mathematical properties and applications. Submetido.

[8] W. Lu \& D. Shi. A new compounding life distribution: the Weibull-Poisson distribution. J. of Appl. Statist., 39 (2012), 21-38.

[9] W. Weibull. A statistical theory of the strenght of material. Ingeniors Vetenskapa Acadamiens Handligar, 151 (1939), 1-45.

[10] W. Weibull. A statistical distribution function of wide applicability. Journal of Applied Mechanics, 18 (1951), 293-296.

[11] W. Weibull. References on Weibull Distribution. Stockholm: FTL A Report, Forsvarets Teletekniska Laboratorium, (1977), 293-296.

[12] W. Alven. "Reliability Engineering by ARINC", Prentice-Hall, Inc., Englewood Cliffs, New Jersey, 1964. 